\title{
Estilos y estrategias de aprendizaje en estudiantes de la Universidad Continental, Huancayo
}

\author{
Styles and strategies of learning in students of the Universidad \\ Continental, Huancayo
}

\author{
Rosario Carrillo Fernández ${ }^{1^{*}}$ \\ ${ }_{1}^{1}$ Universidad Continental, Huancayo
}

\section{RESUMEN}

El objetivo fu determinar la relación que existe entre estilos y estrategias de aprendizaje de los estudiantes de la Facultad de Ciencias de la Salud en la Universidad Continental en Huancayo. Para lo cual se planteó la hipótesis, existe una relación significativa entre estilos y estrategias de aprendizaje de los estudiantes de la Facultad de Ciencias de la Salud en la Universidad Continental Huancayo. La investigación utilizó un diseño descriptivo correlacional y mediante un muestreo probabilístico se obtuvo la muestra de estudio conformada por 231 estudiantes de la Universidad Continental, los datos fueron recopilados mediante la técnica de encuesta con instrumentos validados Chaea de Honey y Munford y Acra abreviado, De la fuente y Justicia adaptadas a educación superior, y validados por juicio de expertos, se utilizó como instrumentos de recolección de datos, cuestionarios aplicados a los estudiantes. Los datos obtenidos fueron procesados haciendo uso de medidas de tendencia central y representada mediante estadígrafos; obteniendo como resultado que los estándares de estilos y estrategias lograron un nivel significativo de acuerdo a la escala de valoración establecida. En conclusión, se ha determinado que existe relación significativa entre estilos y estrategias de aprendizaje de los estudiantes de la Facultad de Ciencias de la Salud en la Universidad Continental Huancayo; con un nivel de significancia de 0,01, Rho $=0,881$ y una " $t$ "calculada $>$ " $t$ " teórica $(28,17>1,96)$ se comprueba la hipótesis planteada.

Palabras clave: Estilo, estrategias, reflexivo, activo teórico, pragmático, habito de estudio, cognitivos, control de aprendizaje, apoyo al aprendizaje.

\begin{abstract}
The objective was to determine the relationship between styles and learning strategies of the students of the Faculty of Health Sciences at the Universidad Continental in Huancayo. For which the hypothesis was raised, there is a significant relationship between styles and learning strategies of the students of the Faculty of Health Sciences at the Universidad Continental Huancayo. The investigation used a correlational descriptive design and by means of a probabilistic sampling the study sample composed of 231 students of the Universidad Continental was obtained, the data were collected by means of the survey technique with validated instruments Chaea de Honey y Munford y Acra abbreviated, De la fuente y Justicia adapted to higher education, and validated by expert judgment, were used as data collection instruments, questionnaires applied to students. The data obtained were processed using measures of central tendency and represented by statisticians; obtaining as a result that the standards of styles and strategies achieved a significant level according to the scale of assessment established. In conclusion, it has been determined that there is a significant relationship between styles and learning strategies of the students of the Faculty of Health Sciences at the Universidad Continental Huancayo; with a level of significance of 0,01 , Rho $=0,881$ and $a$ " $t$ " calculated $>" t$ " theoretical $(28,17>1,96)$ the hypothesis is checked.
\end{abstract}

Keywords: Styles, strategies, reflective, active theoretical, pragmatic, study habits, cognitive control, learning, learning support

Historial del artículo:

Recibido, 05 de noviembre de 2016; aceptado, 8 de enero de 2017; disponible en línea, 25 de junio de 2017

* Doctora en Administración de la Educación, Docente de la Universidad Continental.

Correo: rcarrillo@continental.edu.pe 


\section{INTRODUCCIÓN}

La investigación partió de trabajos realizados en diferentes universidades y están relacionadas con las variables de investigación, concentra en líneas generales un esfuerzo teórico, para brindar en la práctica a la educación una propuesta académica de tal forma, que ayude a resolver el problema de aprendizaje de los estudiantes. Debido a ciertos factores y otros como el desconocimiento de sus estilos y estrategias de aprendizaje, los estudiantes en diversos centros de educación, no tienen una buena asimilación de los conocimientos, que los docentes en sus diferentes áreas les brindan, originándose en ciertos casos, un bajo rendimiento académico. La forma tradicional de enseñar puede que sólo atienda a un estilo y estrategia de aprendizaje. El conocer sobre los estilos y estrategias de aprendizaje nos permite facilitar el proceso de enseñanza aprendizaje, y nos ayuda a reflexionar sobre nuestras funciones y responsabilidades como educadores, ante las necesidades e intereses de nuestros estudiantes ; así algunos antecedentes a nivel internacional se presentan a continuación.

Camarero (2000), desarrolló su investigación en la Universidad de Oviedo, titulada: "Uso de estilos y estrategias de aprendizaje en estudiantes universitarios". Los resultados obtenidos muestran que el estilo de aprendizaje activo se encuentra preferentemente en carreras de humanidades, mientras que el estilo teórico se ubica en mayor proporción en carreras técnicas; con respecto a las estrategias de aprendizaje, se encontró un mayor empleo de estrategias en las especialidades de humanidades y en los alumnos de grados superiores.

Cantú (2004) desarrolló su investigación en Universidad Autónoma de Nuevo León, titulada: "Estilos de aprendizaje y el desempeño académico de los alumnos de arquitectura de la Universidad Autónoma de Nuevo León". Los resultados obtenidos demostraron que el rendimiento académico de los estudiantes expresado por el desempeño (calificación promedio final), es influido, para algunos positivamente y para otros negativamente, por la interacción del estilo de aprendizaje. La autora concluye en términos generales que existe una correlación significativa entre el desempeño académico y el estilo de aprendizaje.

Luengo (2005) desarrolló su investigación en la Universidad de Oviedo, titulada: "Relación entre las predominancias de los estilos de aprendizaje y del alumno y el rendimiento académico en matemáticas". Los autores concluyen que, en la muestra estudiada, existen relaciones significativas entre el rendimiento medio-alto en matemáticas con una mayor predominancia en los estilos de aprendizaje teórico y reflexivo; y se confirma también que el alumnado de cada asignatura optativa conforma un subgrupo homogéneo en cuanto al rendimiento y los estilos de aprendizaje.

Polanco (1996), estudió los estilos de aprendizaje y el desempeño docente en profesores universitarios mejicanos. Con tal fin comparo a dos grupos de docentes, uno formado por el $30 \%$ de docentes evaluados por sus alumnos y otro, compuesto por el $30 \%$ de profesores mejor evaluados, encontró una clara relación entre el estilo divergente y evaluaciones desfavorables de los profesores. Los profesores con procesamiento, activo tendieron a recibir evaluaciones favorables por parte de los estudiantes, mientras que los de procesamiento reflexivo tendieron a ser mal evaluados.

Algunos antecedentes nacionales como el de Chayña (2007), desarrolló su investigación en la Universidad Andina Néstor Cáceres Velásquez de Juliaca, titulada: "Estilos de aprendizaje y su relación con el rendimiento académico de los estudiantes de la FACE UANCV. Facultad de Ciencias de la educación". Los resultados obtenidos muestran que existe una relación significativa entre los estilos de aprendizaje y el rendimiento académico obtenido en los cursos mencionados, por lo cual se concluye que los estilos de aprendizaje y los niveles de rendimiento académico son variables dependientes con un alto grado de correlación.

Así mismo Cárdenas, et al (2005), desarrollaron su investigación en la universidad de Cesar Vallejo de Trujillo, titulada: "Uso de estilos y estrategias en estudiantes universitarios". En los resultados encuentran relación estadística significativa entre la inteligencia general y la observación reflexiva, la conceptualización abstracta y la experimentación activa, pero no con la experiencia concreta.

De igual modo Yacarini (2007), desarrolló su investigación en la Universidad Católica Santo Toribio de Mogrovejo Chiclayo, titulada: "Estilos de aprendizaje y rendimiento académico en estudiantes de la Universidad Católica Santo Toribio de Mogrovejo Chiclayo" los resultados indican que el Estilo de aprendizaje de mayor predominancia en los estudiantes del primer año de estudios generales de la Usat, del tipo reflexivo, seguido por el teórico, pragmatico y activo, y que los estilos de aprendizaje tiene correlación con el rendimiento académico de los estudiantes de la población investigada.

De igual manera lnga (2001), desarrolla su investigación en la Universidad Nacional Federico Villarreal, titulada: "los estilos de aprendizaje y el rendimiento académico en jóvenes universitarios". Los resultados indicaron que el estilo de aprendizaje predominante era el divergente y el menos prevalente, el convergente. 
De igual modo Loret de Mola (2011), desarrollo su investigación en la Universidad Privada los Andes de Huancayo, titulada: "Estilos y estrategias de aprendizaje en el rendimiento académico de los estudiantes de la Universidad Peruana Los Andes. "Se identificaron que los estudiantes utilizan los estilos de aprendizaje de manera diferenciada, siendo de menor utilización el estilo pragmático y de mayor uso el estilo reflexivo; así mismo la estrategia más utilizada es de codificación y la menos usada el apoyo al procedimiento, en cuanto al rendimiento académico los estudiantes se ubican en el nivel bueno. La relación entre las variables de estudio fueron; los estilos de aprendizaje tiene una relación significativa de 0,745 y las estrategias de aprendizaje 0,721 con el rendimiento académico, existiendo una relación positiva significativa según la $r$ de Pearson.

En cuanto a la variable estrategias de aprendizaje Monereo (2014), define que son: Técnicas de aprendizaje y que los recursos varían de acuerdo con los objetivos, contenidos del estudio y aprendizaje de la formación previa de los participantes, posibilidades, capacidades y limitaciones personales de cada quien.

De Zubiría (2006), afirma que existe una diversidad de estrategias, pero, hay una característica común a todas ellas. Así como el atleta desarrolla sus músculos ejercitándolas; sólo es posible desarrollar el pensamiento de los estudiantes colocándoles ejercicios que exijan la puesta en funcionamiento de su capacidad para sintetizar, analizar, abstraer, deducir, en una palabra para pensar. La investigación realizada se justifica ya que es un aporte útil a los esfuerzos por mejorar el nivel de calidad educativa en las carreras de Ciencias de la Salud, de tal manera que sea posible mejorar el aprendizaje de los estudiantes.

El conocimiento de los estilos y las estrategias de aprendizaje de los estudiantes, y la manera en que pueden ser mejorados, resulta beneficioso para los por los siguientes motivos: Para los alumnos, ya que contribuye a que ganen confianza en sí mismos, tengan mayor control sobre el aprendizaje, potencien y expandan su repertorio de estrategias. Para los profesores, es importante tener en cuenta las diferentes maneras de aprender de los estudiantes para que en el diseño de las actividades de enseñanza se contemplen todos los estilos de aprendizaje, los cuales implican diferentes modos de captación de la información y resolución de problemas. Enseñar hábilmente significa poder abarcar todos los estilos y ayudar a ampliar repertorios de estilos y estrategias.

Las variables de investigación parten del fundamento originado en el La Comisión Nacional de Evaluación y Acreditación Universitaria CONEAU referidas a los estándares para el siglo XXI, en cuanto al desarrollo profesional para este siglo que se centra en las tácticas para que los docentes puedan aprovechar oportunidades para integrar habilidades para el siglo $X X I$, herramientas y estrategias de enseñanza dentro de sus prácticas en el aula y ayudarlos a identificar cuáles actividades pueden reemplazar o darles menor importancia ,cultivan la habilidad del docente para identificar estilos y estrategias particulares de aprendizaje en sus estudiantes inteligencias, fortalezas y debilidades estas ayudan a los docentes a desarrollar habilidades en el uso de diferentes estrategias, tales como evaluación formativa, para llegar a una diversidad de estudiantes y para crear ambientes que apoyen en forma diferenciada la enseñanza aprendizaje ante esta realidad latente se planteó el problema ¿̇ué relación existe entre los estilos y estrategias de aprendizaje de los estudiantes de la Facultad de Ciencias de la Salud en la Universidad Continental - Huancayo 2014 ?

\section{MATERIAL Y MÉTODOS}

El diseño de investigación utilizado fue descriptivo correlacional, El diseño de la presente investigación se encuentra en el siguiente esquema:

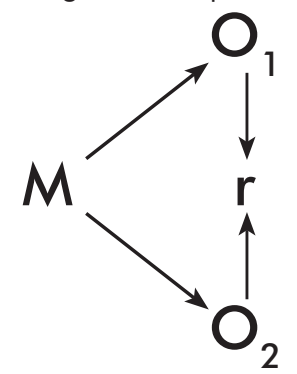

M: Muestra son los 231 estudiantes de la Facultad de Ciencias de la Salud de la Universidad.

En tal sentido para el cálculo de la muestra se aplicó la fórmula del límite central:

$$
n_{0}=\frac{Z^{2} N P Q}{Z^{2} P Q+(N-1) E^{2}}
$$

Donde:

$$
\begin{aligned}
& Z=1,96 \\
& N=577 \\
& P=0,5 \\
& Q=0,5 \\
& E=0,05 \\
& n=231
\end{aligned}
$$

Los instrumentos utilizados fueron la encuesta denominada CHAEA (para estilos de aprendizaje) y ACRA (para estrategias de aprendizaje). Los cuales fueron validados a nivel internacional y nacionalmente mediante juicio de expertos y estadísticamente. 
Tabla 1. Correlación entre Estilos y Estrategias de aprendizaje.

\begin{tabular}{|c|c|c|c|c|}
\hline & Estadigrafos & & $\begin{array}{c}\text { Estilos de } \\
\text { aprendizaje }\end{array}$ & $\begin{array}{c}\text { Estrategia de } \\
\text { aprendizaje }\end{array}$ \\
\hline \multirow[t]{6}{*}{$\begin{array}{l}\text { Rho de } \\
\text { Spearman }\end{array}$} & $\begin{array}{l}\text { Estilos de } \\
\text { aprendizaje }\end{array}$ & $\begin{array}{c}\text { Coeficiente de } \\
\text { correlación }\end{array}$ & 1,000 & $0,881^{* *}$ \\
\hline & & Sig. (bilateral) & 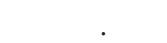 & 0,000 \\
\hline & & $\mathrm{N}$ & 231 & 231 \\
\hline & $\begin{array}{l}\text { Estrategia de } \\
\text { aprendizaje }\end{array}$ & $\begin{array}{c}\text { Coeficiente de } \\
\text { correlación }\end{array}$ & $0,881^{* *}$ & 1,000 \\
\hline & & Sig. (bilateral) & 0,000 & . \\
\hline & & $\mathrm{N}$ & 231 & 231 \\
\hline
\end{tabular}

**. La correlación es significativa al nivel 0,01 (bilateral).

\section{RESULTADOS}

Respecto a la variable estilo de aprendizaje el 18,2\% de estudiantes tienden a un estilo activo; $22,1 \%$ estilo reflexivo; $33,8 \%$ estilo pragmático y $26 \%$ tienden a un estilo teórico.

En relación al resultado de la variable estrategias de aprendizajes, el 26,8 \% de los estudiantes tienden a utilizar la estrategia cognitiva y de control en su aprendizaje; $31,6 \%$ tienden a utilizar la estrategia de apoyo del aprendizaje, y 41,6\% tienden a utilizar la estrategia de hábitos de estudio en su aprendizaje.

Al analizar los resultados en cuanto a la hipótesis general existe una relación significativa entre estilos de aprendizaje y estrategias de aprendizaje de los estudiantes de la Facultad de Ciencias de la Salud en la Universidad Continental Huancayo 2014". Realizando el análisis respectivo podemos señalar que esta relación es directamente en proporción, es decir cuando el estudiantes mayor identifica o tiene en cuenta su estilo de aprendizaje también sus estrategias de aprendizajes aumentan, es una correlación fuerte.

Se concluye estadisticamente que existe relación significativa entre estilos y estrategias de aprendizaje de los estudiantes de la Facultad de Ciencias de la Salud en la universidad Continental Huancayo 2014. con un nivel de significancia de 0,01 , Rho $=0,881$ y una " $t$ "calculada $>$ " $t$ " teórica $(28,17>1,96)$.

\section{DISCUSIÓN}

Existen autores e investigadores que respaldan el resultado, Yacarini (2007) desarrolló su investigación en la Universidad Católica Santo Toribio de Mogrovejo Chiclayo, titulada:" Estilos de aprendizaje y rendimiento académico de los estudiantes de la Universidad Católica Santo Toribio de Mogrovejo". los resultados indican que el estilo de aprendizaje de mayor predominancia en los estudiantes del primer año de estudios generales de la USAT, es del tipo Reflexivo, seguido por el Teórico, Pragmatico y Activo, y que los estrilos de aprendizaje tiene correlación con el rendimiento académico de los estudiantes de la población investigada un $5 \%$ de significancia al 0,73.

El trabajo de Capella (2003), en su investigación titulada "Proceso de enseñanza - aprendizaje de los estudiantes de las diferentes facultades de la Pontificia Universidad Católica del Perú, a partir del conocimiento de los estilos de aprendizaje predominante", el cuestionario utilizando fue el "Cuestionario de Estilos de Aprendizaje" de Honey y Alonso, donde logró determinar que los alumnos presentan un estilo de aprendizaje más reflexivo. Luego se ubican los teóricos, los pragmáticos y los activos. Según sexo identificaron que los varones son más teóricos y más pragmáticos que las mujeres y lo general hay una correlación directa con $r=0,904$.

Fernández (2003) llevó a cabo una investigación que titula "Deficiencias entre estilos de aprendizaje de las alumnas de quinto año de educación secundaria en los colegios católicos privados de la ciudad de Trujillo", del cual concluyó que existen diferencias altamente significativas en los estilos de aprendizaje de las mencionadas alumnas, con un nivel de significancia de 0,03 y que el estilo más predomínate es el pragmático con $48 \%$.

Loret de Mola Garay (2011) en su tesis, titulada: "Estilos y estrategias de aprendizaje en el rendimiento académico de los estudiantes de la Universidad Peruana Los Andes" concluye que identificaron que los estudiantes utilizan los estilos de aprendizaje de manera diferenciada, siendo de menor utilización el estilo pragmático y de mayor uso el estilo reflexivo; así mismo la estrategia más utilizada es de codificación y la menos usada el apoyo al procedimiento, en cuento al rendimiento académico los estudiantes se ubican en el nivel bueno. La relación entre las variables de estudio fueron; los estilos de aprendizaje tiene una relación significativa de 0,745 y las estrategias de aprendizaje 0,721 con el rendimiento académico, existiendo una 
relación positiva significativa según la $r$ de pearson. En consecuencia señalar enfáticamente que los estilos de aprendizaje que presentan los aprendices o estudiantes se ven estrechamente relacionados con multivariables del saber hacer, ser, conocer y actuar. La cual debemos considerarla como una prioridad al momento de nuestra planificación del aprendizaje y/o gestión del aprendizaje.

En cuanto a la variable estrategias de aprendizaje Weinstein y Mayer (1986), mencionan que las estrategias de aprendizaje son conductas o pensamientos que facilitan el aprendizaje. Estas estrategias van desde las simples habilidades de estudio, como el subrayado de la idea principal, hasta los procesos de pensamiento complejo, como el usar las analogías para relacionar el conocimiento previo con la nueva información.

Monereo (2004) las estrategias de aprendizaje son procesos de toma de decisiones (conscientes e intencionales) en los cuales el estudiante elige y recupera, de manera coordinada, los conocimientos que necesita para complementar una determinada demanda u objetivo, dependiendo de las características de la situación educativa en que se produce la acción.

Esto permite explicar que las estrategias de aprendizaje se pueden relacionar con el rendimiento académico, para verificar logros en los estudiantes. Como sea visto para ambas variables la importancia que tienen estos en el procesos de enseñanza aprendizaje dentro y fuera del aula radica en que debemos conocer las tendencias $y / 0$ predominios que se establezcan en nosotros, tanto como estudiantes y docentes, con la única finalidad de saber utilizarla en todo contexto para resolver cualquier conflicto o situación. Pero también debemos advertir que los estilos y las estrategias de aprendizajes no son absolutos ni para toda su vida ya que son rasgos o características donde un sujeto en un determinado espacio temporal actúa de acuerdo a su experiencia adquirida o del contexto en que se encuentre.

Por otra parte, los que hacen la labor de docencia deben tener en consideración los estilos y estrategias de sus estudiantes para que sus sesiones de enseñanza sean significativas y pueda atender las diferencias de sus estudiantes o del grupo a su cargo. Y en cuanto a los directivos o administrativos de la institución deben considerar los estilos y estrategias de aprendizaje para que generen políticas y planes de mejora donde apunten a la mejora de la calidad de aprendizaje enseñanza. Así mismo como aporte la investigación llama la atención a los docentes, porque evidencia algo que en educación se obvia y por consiguiente se omite o se salta es el que las situaciones de aprendizaje son únicas para cada persona implicada; maestros y alumnos que comparten una asignatura, no necesariamente tienen las mismas experiencias y esto si bien es enriquecedor, también muestra la complejidad para el trabajo del estudiante y el docente. Ambos necesitan estar monitoreando sus procesos en conjunto y en lo individual, precisamente por lo distinto de cada experiencia de aprendizaje.

Las clases no pueden planearse asumiendo que hay tiempos muertos; al contrario, exige a los actores estar alerta y revisar el objetivo y los caminos que se están tomando a través de la evaluación del desempeño de las actividades, así como atender a la retroalimentación, para identificar las partes del proceso que se requieren corregir durante el trayecto y no al final.

Se considera que es preciso integrar estrategias para cada fase del aprendizaje y que los estilos favorecen ese

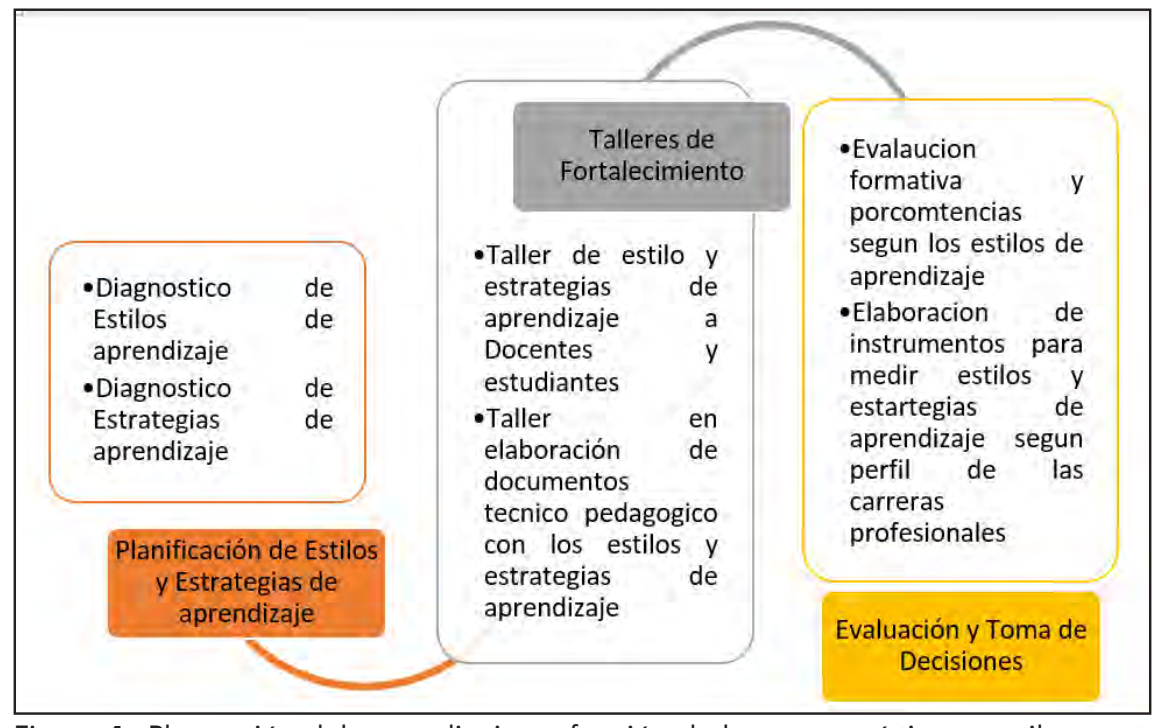

Figura 1. Planeación del aprendizaje en función de las características y estilos y estrategias 
proceso, pero si los alumnos no toman conciencia de que la única forma de aprender es dedicándose justo a eso, solo se quedarán repitiendo superficialmente que si lo saben, pero no van a hacer nada al respecto.

De otro lado se aporta una propuesta de planeación del aprendizaje en función de las características y estilos y estrategias de los estudiantes (figura 1).

\section{REFERENCIAS BIBLIOGRÁFICAS}

Camarero, F.J. (2000) Estilos y estrategias de aprendizaje en estudiantes universitarios. Tesis Doctoral. Universidad de Oviedo.

Cantú H., I.L. (2004) El Estilo de Aprendizaje y la Relación con el Desempeño Académico en Los Estudiantes de Arquitectura de la UANL, en: Ciencia UANL, Vol. VII, No. 1. Monterrey, N.L., México.

Cantú, H. I. L. (2004) "El estilo de aprendizaje y la relación con el desempeño académico de los estudiantes de arquitectura de la UANL". Revista Ciencia UANL, 7 (1), 72-79.

Cárdenas, K; et al.(2005) Inteligencia y estilos de aprendizaje en alumnos universitarios ingresantes. Revista de Psicología Universidad Cesar Vallejo, Facultad de Humanidades, escuela de Psicología, Vol. 7, pp.61-66.95

Cárdenas, K \& Vera, M. (2003).Estilos de aprendizaje y cohesión familiar de los alumnos y docentes de formación básica- letras en el periodo 2002 -l de la Universidad Cesar Vallejo de Trujillo. Revista de Psicología Universidad Cesar Vallejo, Facultad de Humanidades, escuela de Psicología, Vol. 5, pp.108-119.

Capella, J., \& Vargas, J.(2003). Serie: Cuadernos de educación. Estilos de Aprendizaje. Lima: Fondo Editorial Pontificia Universidad Católica del Perú.

Chayña G., M. (2007) Estilos de Aprendizaje y su Relación con el Rendimiento Académico en los Estudiantes de la FACE-UANCV. Facultad de Ciencias de la Educación de la Universidad Andina Néstor Cáceres Velásquez. Juliaca, Perú.
De Zubiría, M. \& De Zubiría, J. (2006). Biografía del pensamiento, estrategias para el desarrollo de la inteligencia. Colombia: Cooperativa Editorial Magisterio.

Fernández, J. \& Villanueva (2003) Diferencia entre Los Estilos de aprendizaje de las alumnas del 5to Año de Secundaria de los Colegios católicos Privados de la Ciudad de Trujillo. Tesis de maestría, Universidad Cesar Vallejo, Perú

Inga, J. (2001). Estilos de Aprendizaje y Rendimiento académico en universitarios. Revista Científica Wiñay Yachay. Vol. 2, No.1, pp. 93-98.

Luengo G. (2005). Relación Entre los Estilos de Aprendizaje, el Rendimiento en Matemáticas y la Elección de Asignaturas Optativas en Alumnos de Enseñanza Secundaria Obligatoria (E.S.O.). En: Unión. Revista Iberoamericana de Educación Matemática, No. 3. Madrid, España. pp 25-46. Tesis doctoral

Loret de Mola. (2011) Estilos y estrategias de aprendizaje en el rendimiento académico de los estudiantes de la Universidad Peruana los Andes. Tesis para optar el grado de magister UPLA. Huancayo Perú.

Monereo, C. (2004). Estrategias de Enseñanza y aprendizaje. Formación del profesorado aplicación en la escuela. Graó. Barcelona.

Polanco, R. (1996). El Estilo de ajuste como predictor del desempeño docente en profesores de áreas administrativas-sociales y de ingenierías. II Jornadas de innovación en la enseñanza de las ingenierías. Tesis Doctoral Madrid.

Weinstein, C. \&. Mayer R .(1986) The teaching of learning strategies. En Wittrock, M. C. Handbook of Reserach of Teaching. McMillian. Nueva York.

Yacarini M., \& Gómez C., J. (2007) Estilos de Aprendizaje y Rendimiento Académico en Estudiantes de la Universidad Católica de Santo Toribio de Mogrovejo 